\title{
Autobiographic Knowledge for Believable Virtual Characters
}

\author{
Wan Ching Ho, Scott Watson \\ Adaptive Systems Research Group \\ School of Computer Science, University of Hertfordshire \\ College Lane, Hatfield, Hertfordshire, AL10 9AB, UK \\ [W.C.Ho | S.E.J.Watson] @herts.ac.uk
}

\begin{abstract}
It has been widely acknowledged in the areas of human memory and cognition that behaviour and emotion are essentially grounded by autobiographic knowledge. In this paper we propose an overall framework of human autobiographic memory for modelling believable virtual characters in narrative story-telling systems and role-playing computer games. We first lay out the background research of autobiographic memory in Psychology, Cognitive Science and Artificial Intelligence. Our autobiographic agent framework is then detailed with features supporting other cognitive processes which have been extensively modelled in the design of believable virtual characters (e.g. goal structure, emotion, attention, memory schema and reactive behaviour-based control at a lower level). Finally we list directions for future research at the end of the paper.
\end{abstract}

\section{Introduction}

It is widely held that a human's personality and problem-solving abilities are strongly influenced by his/her past experience. Inspired by psychological research in human memory, which states that autobiographic memory is a specific kind of episodic memory that may develop in childhood [1], Dautenhahn introduced autobiographic agents; agents which are embodied and situated in a particular environment (including other agents), and which dynamically reconstruct their individual history (autobiography) during their lifetimes [2]. Since autobiographic memory particularly focuses on meaningful and significant events for intelligent agents, it can also be used for 1) synthesising agents that can behave adaptively [3] and in socially intelligent ways [4] and 2) designing agents that appear believable and acceptable to humans.

Research in believable virtual agents has also utilised the human cognitive memory model [5] from the field of cognitive science and psychology, such as the study in synthetic vision for autonomous virtual humans [6]. Strategies for memory storage are usually divided into Sensory, Short-term and Long-term memories according to the rehearsal process and the retaining length of time for an item to be remembered. However, imposing a human memory model on virtual agents without taking its real cognitive abilities into account would meet various limitations. For example, only simple items can be remembered, agents are not able to decide which item is more important to itself or to other agents, and the difficulty in creating temporal sequences of items in memory. 
In addition to memory models, modelling other human cognitive processes has also been a popular research direction in developing architectures for IVAs (intelligent virtual agents) in recent years. For example, general computational models of human emotion for IVAs have attracted a lot of attention. Marsella and Gratch [7] utilised appraisal theory from psychology to model emotion for believable characters that perform in various applications. Their approach has also been used and enhanced by the VICTEC project team [8] to create believable synthetic characters acting in antibullying dramas.

Marsella and Gratch stated that appraisal variables enable agents to characterize the significance of events from the individual's perspective as the interpretation of each event is altered by an agent's own beliefs, desires and intentions, and past events. Here we are interested in establishing links between agents' current cognitive processes (e.g. goal formulation and emotion) and their past experiences autobiographic memory.

Furthermore, research findings from psychologists Conway [9, 10 and 13] and Healy and Williams [11] provide rich evidence to show how autobiographic memory grounds other cognitive processes which operate in everyday human life. These findings have inspired us to develop an overall framework for an agent architecture supported by autobiographic knowledge.

\section{Autobiographic Memory Research in Psychology, Cognitive Science and AI}

"In the past, it (autobiographic memory) has usually been conceived of in terms of childhood (or infantile) amnesia, the phenomenon, first identified by Freud and familiar to all who reflect on it, that memories for events from the early years of our lives - before about 3 to 4 years - are not available to adult consciousness, although many memories from later childhood usually are easily called up.” [1, page 8]

\section{Autobiographic Memory Research in Psychology and Cognitive Science}

Autobiographic memory is a specific kind of episodic memory that contains personally significant and meaningful experiences. Two features of autobiographic memory are generally defined and accepted by researchers in psychology, as pointed out by [12]:

- Autobiographic memories are mental constructions of the self.

- They very often feature imagery whilst simultaneously containing abstract personal knowledge. 


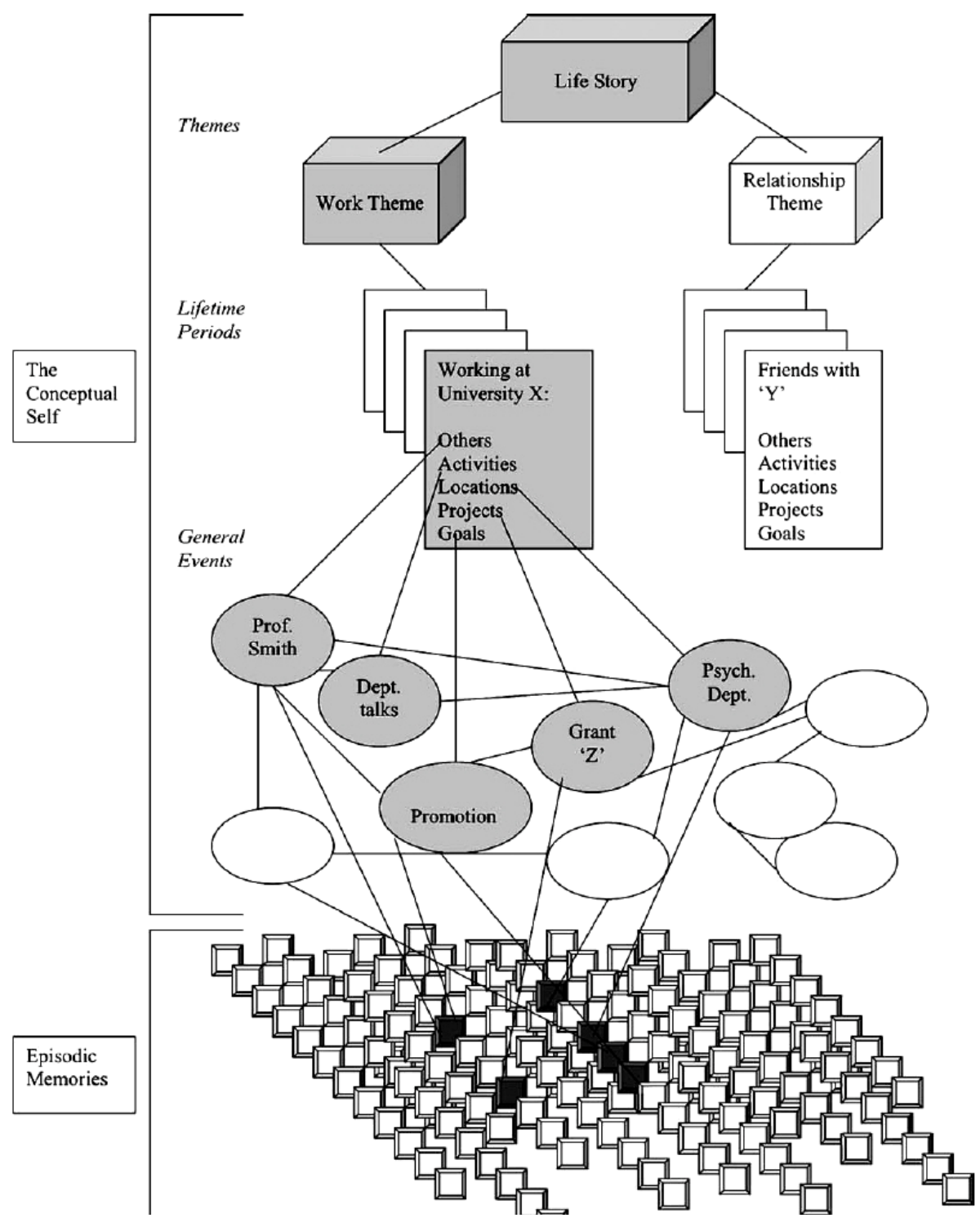

Figure 1.1 Knowledge structures in autobiographic memory. Extracted from [13, page 609].

Figure 1.1 extracted from [13] shows the hierarchical knowledge structures in the autobiographic knowledge-base in which Conway indicates that lifetime periods may themselves be thematically linked together (he uses work and relationship themes from his own past as examples in this figure). Conway shows that the conceptual self of a human being is composed and shaped by frequently reconstructing general events from retrieved episodic memories - these general events eventually form different life periods and themes in someone's life story. 
From the perspective of considering humans as social beings, Nelson (a developmental psychologist) carried out her investigations into how children develop their own autobiographic memory, and suggests that the primary function of autobiographic memory is to share experiences with other people [1]. Other studies in psychological and cognitive development have also pointed out the cognitive, as well as social, functions of autobiographic memory as underlying all of human story telling and history-making narrative activity. Kelly and Dickinson studied the autobiographic accounts of the experience of chronic illness and suggested that "self is not a biologistic or psychologistic thing, rather self is autobiographic narrative." [14, page 224]. Neisser [15] suggests that recalling an experienced event from autobiographic memory is not a matter of reviving a single record but of moving appropriately among nested levels of structure. As the rehearsal process takes place, the memory structure is fundamentally changed by this recall. Moreover, a recall is always reconstructing an event by some other similar events of experience: Specifically, goals and current situations of the recall affect how much detail can be obtained from the event which is being remembered [15].

Self-schemata for maintaining the integrity and gist of past life events have been discussed by Barclay [16]. He argued that events in autobiographic memories change over time since new events occur and many life experiences become repetitious, making any single event indistinguishable from related happenings. Thus inaccurate remembering and forgetting take place because of the merging of episodic memories into more generic event categories representing the semantic features of everyday activities.

The importance of autobiographic memory to everyday life is further supported by findings from clinical studies. Healy and Williams [11] found that PTSD (Post Traumatic Stress Disorder) and OCD (Obsessive-Compulsive Disorder) patients tend to retrieve over-general memory contents in order to avoid emotional depression when remembering any specific episode. They stated, however, that this phenomenon influences patients' memory encoding and retrieval in their autobiographic memory over a long period of time. As a result, patients' working memories are decayed by the frequent and active forgetting of details of depressing episodes - thus also leading to a reduction in their general problem solving skills. This is significant as it demonstrates the negative effects which can occur when autobiographical memory goes wrong.

\section{Applying Autobiographic Memory in Agent Research}

Dautenhahn and Coles [17] carried out the first set of pioneering experiments on autobiographic agents, in which Situation-Action-Situation triplets were used as the core of the agents' autobiography. This work compared trajectories and lifetimes of purely reactive (sensory-driven) and post-reactive (memory-driven) control agents. Results from the experiments show that autobiographic memory, when embedded in the control architecture, can effectively extend an agent's lifetime.

Studies from [18] and [19] implemented finite-state autobiographic memory into the basic control architecture for a Purely Reactive agent to study autobiographic agents from the bottom-up approach and Artificial Life perspective. Different static virtual environments with resource allocations are created for either single or multiple agents to 'survive' in. In order to find out the location of resources by using local 
information from sensory data, autobiographic agents are able to remember their previous action sequences for going back to the particular resources. Taken together, these studies show that autobiographic agents and agents that share experiences outperform purely reactive agents in surviving in the static environments (this is true for both single agent and multi-agent experiments). After these early studies on basic memory architectures for narrative autobiographic agents, recent studies from [20] and [21] with enhanced computational autobiographic memory architectures and a complex and dynamic environment again show that autobiographic memory can prove beneficial - as indicated by the increased lifespan of autonomous, autobiographic, minimal agents. Furthermore, higher communication frequency brings better group performance for Long-term Autobiographic Memory agents in multi-agent experiments. It is evident that embedding narrative story-telling in LTM control architecture as an additional communication feature helps agents to be more adaptive in coping with different environmental dynamics.

Agent architectures with Categorical Long-term Autobiographic Memory which remember both positive and negative events are developed and investigated in [22]. Experimental results from the study show that it is advantageous for agents to 1) categorise the remembered events for efficient memory retrieval, and 2) remember various negative events so that they can avoid making same mistakes again.

\section{Computational Framework for Autobiographic Agents}

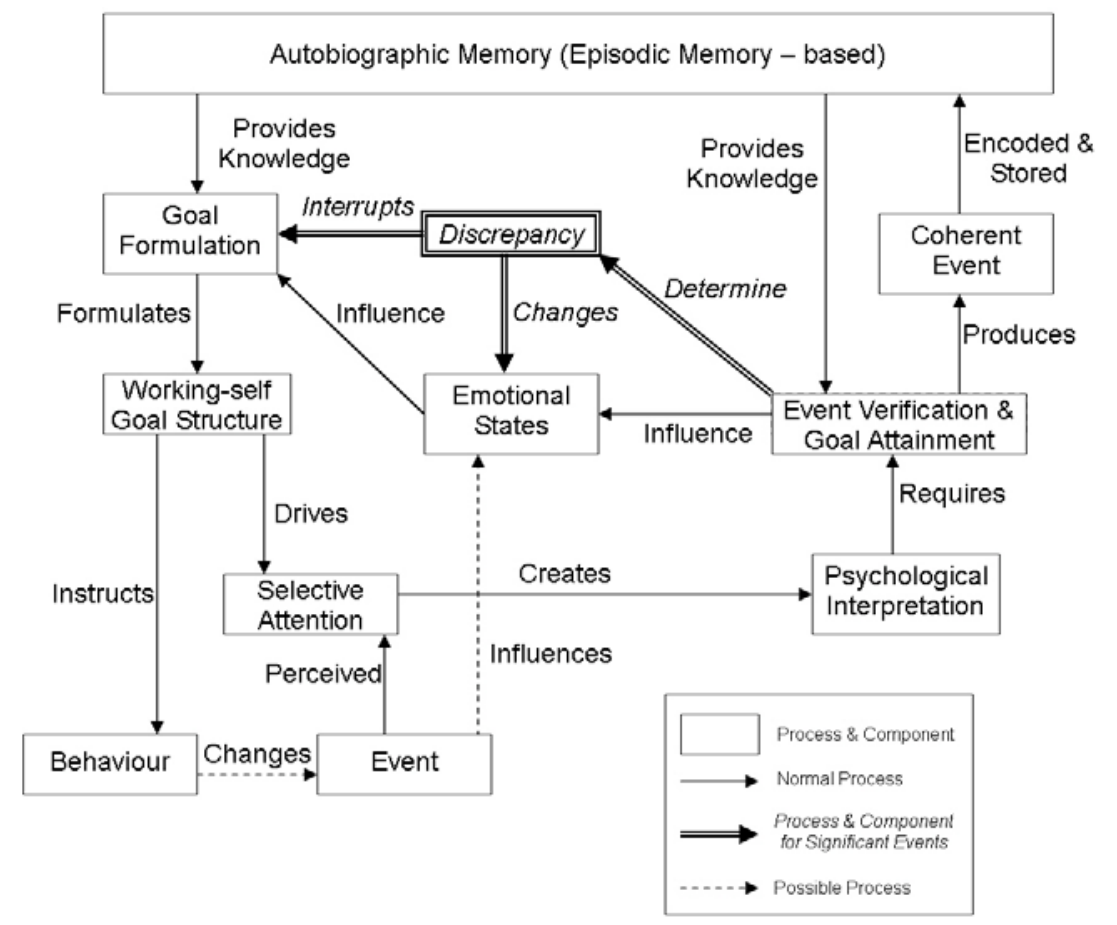

Figure 2.1 Computational framework for autobiographic agents showing how autobiographic knowledge supports other cognitive processes 
Figure 2.1 illustrates our overall framework of autobiographic agents. Although many human cognitive processes we propose in this framework have been modelled by researchers working on believable virtual characters, such as attention, emotion and goal structure; many links between these cognitive processes and agents' knowledge base which contains agents' past experiences are still missing.

In this section we first focus on features of autobiographic knowledge and the components which are associating with it, as follows:

- Knowledge representation in autobiographic memory, based on episodic memory.

- Goal structure, emotion, and attention processes, in supporting or being influenced by, other cognitive processes.

Furthermore, we are also interested in exploring the potential of autobiographic agents for character-based narrative story-telling systems. How autobiographic narrative is generated from our agent framework is discussed at the end of this section.

\section{Knowledge Representation in Autobiographic Memory}

Information remembered in human autobiographic memory is based on event reconstructions on top of episodic memory. This process of event reconstruction provides memory rehearsal to consolidate frequently remembered information and elaborates cues for memory retrieval dealing with the current situation [10]. Computational autobiographic memory architectures supporting real-time event reconstructions for Artificial Life agents have been implemented in [20] and [21]. Experimental results from both papers show agents with Long-term Autobiographic Memory architectures have higher adaptivity when surviving in a complex and dynamic environment, compared to Reactive and Short-term Memory architectures.

As described in the previous section, literature from Psychology and Cognitive Science indicate that human autobiographic memory contains meaningful personal experiences - information that constructs the 'self' and shapes someone's 'personality'. The significance of each event remembered in agents' autobiographic memory plays an important role during processes of memory encoding and retrieval. Previous studies have shown that, having recognised the event significance, agents are able to re-experience a typical action sequence of the most meaningful event in their memory; therefore they outperform other types of agents, as shown in studies [20-23].

Autobiographic memory contains coherent personal events. New experiences must therefore be encoded into autobiographic memory in a similarly coherent way. In this way, experiences are verified with existing knowledge, maintaining the agent's autobiographic 'self'. In the case that a novel situation is experienced, the agent may lack relevant autobiographic knowledge, and will have to fall back on more instinctive behaviours.

It is worth pointing out here the difference in roles played by semantic knowledge in contrast with autobiographic knowledge. Semantic knowledge provides objective information about the world and is entirely passive; whereas autobiographic knowledge includes subjective representations of events that are actively encoded into memory [37]. 


\section{Goal Structure, Emotion, and Attention Processes}

The goal structure in our model follows that of Conway [13], in that it is designed to form an iterative loop whose purpose is to reduce the discrepancy between desired and actual goal states. In performing this discrepancy reduction, behaviour is regulated. Goals (and their respective sub-goals) emerge from autobiographic knowledge and guide selective attention and the behavioural processes necessary to perpetuate the current goal structure. In order to ensure this structure is maintained, the autobiographic knowledge base also continuously monitors events and verifies the current goal attainment. In this way, the goal structure directs behaviour in a manner which compliments the agent's previous understanding of the situation. In turn, this verification process will lead to the encoding and further consolidation of a generic schema-based memory (e.g. Barclay [16]) - presumably in order to reduce the quantity of information that actually has to be stored and to facilitate a more cohesive 'self'. In summary, the autobiographic knowledge-base is instrumental in the generation and maintenance of a goal structure, thus providing a cohesive narrative for the agent to follow.

This perpetual goal structure may be interrupted, however, most likely by causes outside of the agent's control. In the case that the desired goal is not attained, or the current situation cannot be verified, a large discrepancy (between desired and actual goal states) can occur. This discrepancy will interrupt the normal goal-formulation process and will also negatively affect the agent's emotion.

A new set of goals will need to be formed in order to cope with the current situation and to reduce this discrepancy as quickly as possible - again drawing upon information contained in the autobiographic knowledge-base. If the discrepancy is so large that the autobiographic knowledge-base contains no information relevant to the formulation of appropriate goals, the agent will be forced to fall back on emotionbased coping strategies and (in extreme cases) instinctive behaviours.

For this reason it is also necessary to model the role that emotion plays on the goal formulation process. Recent evidence from psychophysiological studies is consistent in the finding that events may generate emotions quickly (within 100-300ms of stimuli) and without conscious processing - a pattern which suggests 'pre-attentive' processing of emotional valence (see Compton [24] for a recent review). Furthermore, it is becoming apparent (under a number of methodological paradigms) that this preattentive activation of emotions can guide selective attention via both high-level cortical pathways as well as low-level routes through the amygdala. Taken together, this pattern of results suggests that emotion influences the normal goal formulation process (by shaping sub-goals which govern selective attention), but can also generate coping strategies when the autobiographic knowledge-base is found lacking. Whether successful or unsuccessful, these novel coping strategies are themselves encoded into the autobiographic knowledge-base thus creating new schemas which can be used to inform future goal formulation.

A major advantage of this model over previous appraisal models, such as those based on the OCC (Ortony, Clore and Collins) model, is that it conceives a perpetual schema-based, autobiographically-driven narrative 'self' which also allows the agent to learn from the experience of novel situations. Without autobiographic memory an agent may be doomed to repeat actions that have previously been unsuccessful. However, with autobiographic memory implemented an agent is free to learn from its 
mistakes and generate new coping strategies. Therefore the OCC model would benefit from the inclusion of the history function afforded by autobiographic memory [38].

\section{Supporting Narrative Story-telling}

Research in narrative intelligence aims to develop agents which can have the capacities of story-awareness, story-telling and historical grounding. Concerned with building this kind of narrative agent, the area has investigated various directions such as interactive drama or story-telling [29-32], social understanding [33-35] and narrative in virtual environments [36]. Researchers have generated fruitful ideas that enhance both story-telling abilities and believability of narrative agents which interact with human users.

Narrative agents are usually pre-programmed either with temporal and structured stories or with simultaneously selecting story sequences from a large story database when they are interacting with human users in story-telling systems. Therefore they can take advantage of mechanisms used in natural historically grounded systems (Nehaniv [27]) and enhance the friendliness of these systems. While appreciating the success narrative agents bring to story-telling systems or software interfaces, the investigation of how agents themselves can benefit from autobiographic narrative using their existing experiences to interpret a new story - is missing in the Narrative Intelligence research field. A new story is defined here as an agent perceiving a new event itself or receiving a story from other another agent through communication. An agent should then be able to form a unique psychological interpretation of this story by drawing upon their own existing autobiographic knowledge.

Humans are naturally expert in both understanding and producing narrative as we can express almost anything without necessarily using natural language but simply by showing appropriate gestures or facial expressions. In contrast, it is very difficult for virtual agents to have the same quality of result. This leads to the problem of behavioural incoherence - human observers often cannot understand why agents behave as they do. This phenomenon is described by Sengers as 'schizophrenia' [25]. She argues that architecture for narrative agents should be able to structure behaviour to be comprehensible as narrative. This behavioural coherence which originated with the life story of human beings [28] is particularly important for believable agents in narrative story-telling environments. Therefore, except system interfaces which show the agents' 'intentions' to the user [23], behavioural patterns expressed by narrative virtual agents have to be generated from 1) reconstructing their past experiences in autobiographic memory and 2) existing knowledge of the surrounding agents and the environment.

Another main research issue in character-based narrative story-telling system, i.e. role-play dramas or games, is how to provide good 'actors' - agents that react to different situations in stories with appropriate emotional expression [23]. We argue that taking a long-term character development approach, rather than setting a fixed group of parameters for instructing agents to 'act' in a particular situation, is more realistic and flexible to shape agents' 'personality' in a story and to determine their emotion in a given situation by allowing them to have their own autobiographic knowledge. This approach thus 1) allows for the creation of agents which are 'born with' certain types of personality for role-play stories, and 2) supports long-term 
development and learning of virtual actors as they gain new experience from acting in each new situation.

Furthermore, the potential of autobiographic agents to create stories from their own experiences and understand stories from others enhances the endurance of events remembered in their autobiographic memory. Nelson [1] pointed out that in addition to the function of language, humans sharing memories with other people can be seen as narrative story-telling that performs a significant social-cultural function, and both these two functions explain why personal autobiographic memories continue to persist during their lifetime.

\section{Conclusion and Future Work}

In this paper, we propose a framework to model human autobiographic memory for believable virtual characters. From research findings in Psychology and Cognitive Science, we argued that autobiographic knowledge is necessary for virtual agents in terms of learning from past experiences and long-term developments. We have also discussed our previous studies in Artificial Life autobiographic agents. On a behavioural level, they can be perceived as a foundation for investigating how autobiographic memory enhances agents' adaptivity and believability. Similarly, other important cognitive processes, such as goal formulation and emotion lead agents to perform appropriate behaviours in a given situation, should also be grounded by autobiographic knowledge.

Further development for the currently proposed autobiographic knowledge framework is to implement it into an agent-based emergent narrative system, which will be developed in the EU Framework 6 funded project 'eCIRCUS' (Education through Characters with emotional-Intelligence and Role-playing Capabilities that Understand Social interaction).

The first step is to specify the knowledge representation in episodic memory in order to reconstruct general events, themes and possibly life periods for forming agents' autobiographic 'self', based on the prototype created for Artificial Life agents in [22] and [23]. Memory encoding and retrieval mechanisms also need to be developed and validated for guiding agents' behaviour, planning, and changes in emotion. The second step would be to create semantic memory from accumulated repetitive episodic events. Semantic memory, as utilized in many conventional AI learning approaches, may allow agents to take advantage of prior knowledge in perceiving and organizing both the physical can social world around them.

Human memory is fallible. False memories can constructed, while true memories may be incorrectly recalled or even forgotten. While these negative characteristics of human autobiographic memory are important (and could be modelled for academic purposes), we do not believe that users currently interact with agents for long enough periods of time to require this be included.

In addition to the implementation of the whole autobiographic memory framework for creating believable characters, the component of autobiographic memory can be separately integrated into other agent cognitive architectures, such as [7] and [8]. In this case, links between agents' autobiographic memory and other cognitive processes are critical in establishing 1) the generation of believable behaviour and 2) agents' learning ability and long-term development. 


\section{Acknowledgements}

This paper is supported by the eCIRCUS project carried out with the provision of the European Community in the Framework VI Programme.

\section{References}

1. Nelson, K. (1993) The Psychological and social origins of autobiographical memory. Psychological Science, 4:7-14.

2. Dautenhahn, K. (1996) Embodiment in animals and artefacts, Proc. AAAI FS Embodied Cognition and Action, AAAI Press, Technical report FS-96-02, 27-32.

3. Nehaniv, C. L. and Dautenhahn, K. (1998) Embodiment and memories - algebras of time and history for autobiographic agents, 14th European Meeting on Cybernetics and Systems Research, Embodied Cognition and AI symposium, pp. 651-656.

4. Dautenhahn, K. (1999), Embodiment and interaction in socially intelligent lifelike agents, in C. L. Nehaniv (ed.), Computation for Metaphors, Analogy and Agent, Vol. 1562 of Springer Lecture Notes in Artificial Intelligence, Springer, pp. 102-142.

5. Norman, D. A. and Bobrow, D. G. (1975) On the role of active memory processes in perception and cognition, in C. N. Cofer (ed.), The Structure of Human Memory, W. H. Freeman and Company, pp. 114-132.

6. Peters, C. and O'Sullivan, C. (2002) Synthetic vision and memory for autonomous virtual humans, Computer Graphics Forum 21(4), 743-753.

7. Marsella, S., Gratch, J. (2003) Modelling coping behaviour in virtual humans: Don’t worry, be happy, Proceedings of Second International Joint Conference on Autonomous Agents and Multiagent Systems, ACM Press, 2003.

8. VICTEC (2005) Virtual ICT with empathic characters, http://www.victec.net/. Last accessed 29-03-2006.

9. Conway, M. A. (1996) Autobiographical memories and autobiographical knowledge, in D. C. Rubin (ed.), Remembering our past: Studies in autobiographical memory, Cambridge Univ. Press, Cam0bridge, UK, pp. 67-93.

10. Conway, M. A. and Pleydell-Pearce, C. W. (2000) The construction of autobiographical memories in the self memory system, Psychological Review 107, 261-288.

11. Healy, H., Williams, J. M. G. (1999) Autobiographic Memory, Handbook of Cognition and Emotion, pp. 229-242.

12. Conway, M. A., Pleydell-Pearce, C. W. and Whitecross, S. E. (2001) The neuroanatomy of autobiographical memory: A slow cortical potential study (SCP) of autobiographical memory retrieval, Memory and Language, 45, pp. 493-524.

13. Conway, M. A. (2005), Memory and the self, Journal of Memory and Language 53, pp. 594-628.

14. Kelly, M. P. and Dickinson, H. (1997) The narrative self in autobiographical accounts of illness, The Sociological Review, 45(2), pp. 254-278.

15. Neisser, U. (1986) Nested structure in autobiographical memory, in D. C. Rubin (ed.), Autobiographical Memory, Cambridge University Press, Cambridge, UK, pp. 71-88.

16. Barclay, C. R. (1986) Schematization of autobiographical memory, in D. C. Rubin (ed.), Autobiographical Memory, Cambridge University Press, Cambridge, USA, pp. 82-99.

17. Dautenhahn, K. and Coles, S. (2001) Narrative intelligence from the bottom up: A computational framework for the study of story-telling in autonomous agents, The Journal of Artificial Societies and Social Simulation (JASSS), 4(1), pp. 1-15. http://jasss.soc.surrey.ac.uk/4/1/1.html (Last accessed 16-09-2005). 
18. Ho, W. C., Dautenhahn, K. and Nehaniv, C. L. (2003) Comparing different control architectures for autobiographic agents in static virtual environments, Intelligent Virtual Agents 2003 (IVA 2003), Springer LNAI, pp. 182-191.

19. Ho, W. C., Dautenhahn, K., Nehaniv, C. L. and te Boekhorst, R. (2004) Sharing memories: An experimental investigation with multiple autonomous autobiographic agents, IAS-8, 8th Conference on Intelligent Autonomous Systems, IOS Press, Amsterdam, NL, pp. 361-370.

20. Ho, W. C., Dautenhahn, K. and Nehaniv, C. L. (2005) Autobiographic agents in dynamic virtual environments - performance comparison for different memory control architectures, Proceedings of IEEE Congress on Evolutionary Computation - Special Session: Artificial Life, pp. 573-580.

21. Ho, W. C., Dautenhahn, K. and Nehaniv, C. L. (submitted) A bottom-up study in autonomous agents with autonomous memory and narrative storytelling in a dynamic virtual environment, Cognitive Systems Research.

22. Ho, W. C., Dautenhahn, K. and Nehaniv, C. L. (2006) A study of episodic memory-based learning and narrative structure for autobiographic agents, Proceedings of Adaptation in Artificial and Biological Systems, 3, AISB 2006 conference, pp. 26-29.

23. Ho, W. C., (2005) Computational memory architectures for autobiographic and narrative virtual agents, Unpublished $\mathrm{PhD}$ Thesis, University of Hertfordshire.

24. Compton, R.J. (2003) The interface between emotion and attention: A review of evidence from psychology and neuroscience, Behavioural and Cognitive Neuroscience Reviews, 2(2), pp. 115-129.

25. Sengers, P. (2003) Narrative and schizophrenia in artificial agents, in M. Mateas and P. Sengers (eds), Narrative Intelligence, Amsterdam: John Benjamins, pp. 259-278.

26. Louchart, S., Aylett, R., Enz, S. and Dias J. (2006) Understanding emotions in drama, a step towards interactive narrative, Proceedings of Adaptation in Artificial and Biological Systems, 3, AISB 2006 conference, pp. 38-44.

27. Nehaniv C. L. (1999) Story-telling and emotion: Cognitive technology considerations in networking temporally and affectively grounded minds, Third International Conference on Cognitive Technology: Networked Minds (CT'99), Aug. 11-14, 1999 San Francisco/Silicon Valley, USA, pp. 313-322.

28. Linde, C. (1993) Life Stories: The Creation of Coherence, Oxford University Press.

29. Mateas, M. (1999) An Oz-centric review of interactive drama and believable agents, Artificial Intelligence Today pp. 297-328.

30. Mateas, M. and Stern, A. (2002) Architecture, authorial idioms and early observations of the interactive drama facade, Technical report, School of Computer Science, Carnegie Mellon University. CMU-CS-02-198.

31. Stern, A. (2003) Virtual babyz: Believable agents with narrative intelligence, in M. Mateas and P. Sengers (eds), Narrative Intelligence, John Benjamins Publishing, pp. 215-227.

32. Cavazza, M., Martin, O., Charles, F., Mead, S. J. and Marichal, X. (2003) Interacting with virtual agents in mixed reality interactive storytelling, Intelligent Virtual Agents 2003 (IVA 2003), Springer LNAI, pp. 231-235.

33. Dautenhahn, K. and Nehaniv, C. L.: 1998, Artificial life and natural stories, International Symposium on Artificial Life and Robotics (AROB III), Vol. 2, Beppu, Oita, Japan, pp. 435-439.

34. Dautenhahn, K. (2002) The origins of narrative in search for the transactional format of narratives in humans and other social animals, Cognition and Technology: Co-existence, Convergence, Co-evolution (IJCT) pp. 97-123. John Benjamins Publishing Company.

35. Dautenhahn, K. (2003) Stories of lemurs and robots - the social origin of storytelling, in M. Mateas and P. Sengers (eds), Narrative Intelligence, John Benjamins Publishing, pp. 63-90.

36. Aylett, R. (1999) Narrative in virtual environments: Towards emergent narrative, Proc. Narrative Intelligence, AAAI Fall Symposium 1999, AAAI Press, pp. 83-86.

37. Fentress, J. \& Wickham, C. (1992) Social memory - new perspectives on the past. Blackwell. 


\section{Wan Ching Ho, Scott Watson}

38. Bartneck, C. (2002). Integrating the OCC model of emotions in embodied characters. Proceedings of the workshop on virtual conversational characters: applications, methods, and research challenges, Melbourne. 